\title{
MATHEMATICAL ANALYSIS AND SIMULATION OF \\ A COUPLED NONLINEAR FLUID STRUCTURE INTERACTION MODEL WITH APPLICATIONS TO ANEURYSMS
}

\author{
MANAL BADGAISH ${ }^{1}$, JENG-ENG LIN ${ }^{2}$, \\ AND PADMANABHAN SESHAIYER ${ }^{3}$ \\ ${ }^{1}$ Department of Mathematical Sciences \\ Umm Al-Qura University \\ Makkah, P.O. Box 715, KSA \\ ${ }^{2,3}$ Department of Mathematical Sciences \\ George Mason University \\ Fairfax, VA 22030 USA
}

\begin{abstract}
In this work, we will present a mathematical model that describes a coupled fluid-structure interaction between the arterial wall, the blood flow inside the wall and the cerebral spinal fluid outside the wall with applications to intracranial saccular aneurysms. The governing system of differential equations includes a nonlinear power-law fluid equation coupled with a nonlinear elasticity equation describing the wall in conjunction with blood pressure that is modeled via a Fourier series. The thrust of this work involves the analysis and simulation of the associated mathematical model using classical differential equation techniques. Besides proving existence and uniqueness of the solution to the related system, analytical expressions for the associated traveling wave solutions for the governing nonlinear differential equation is also derived for a special class of problems that is biologically tractable.
\end{abstract}

AMS Subject Classification: 65M12, 65P 40

Received: June 13, 2017; Accepted: October 31, 2018;

Published: November 13, 2018 doi: 10.12732/caa.v22i4.10

Dynamic Publishers, Inc., Acad. Publishers, Ltd. http://www.acadsol.eu/caa 


\section{INTRODUCTION}

Intracranial saccular aneurysms are focal dilatation located in the arterial wall. These lesions are soft tissues filled with the blood and surrounded by cerebral spinal fluid. Due to a variety of bio-mechanical factors, the growing aneurysm may leak or rupture which can lead to a neurological disorder or death. About $80 \%$ to $90 \%$ of ruptured aneurysm may cause death [27]. Employing a surgical procedure would be a choice to avoiding that. Typical procedures include clipping which is an invasive approach where a small metal clip is inserted in the base of the aneurysm to prevent it from rupturing. An alternative procedure is endovascular repair where the surgeon inserts a wire to occlude the inside of the aneurysm hence preventing the blood to enter the aneurysm. However, such procedures often involves potential risks including behavior changes due to neurological injury and potential for blood clots, brain swelling, stroke and seizures [14].

Several researchers believe that the elastodynamics behavior of the arterial wall interacting with the blood flow is one of the main reasons for aneurysm rupture $[10,22,16]$. Over the last two decades there has been progress in the development of mathematical models to understand the rupture of aneurysms (see [17, 18, 19, 25] and references therein). Due to the coupled multi-physics nature of the problem, there have been several related fluid structure interaction models that have been studied extensively [20, 24, 6, 15]. These models describe the interaction of blood pressure, arterial wall, and Cerebral Spinal Fluid (CSF). In [24], the blood pressure was modeled via a Fourier series assuming blood flow is pulsatile whereas the CSF was modeled using a simplified one-dimensional compressible Euler equation for inviscid flow and negligible nonlinear effects. Recently, this model was extended to incorporate the complex biological structure of the arterial wall by including the growth and remodeling effects of two main constituents, elastin and collagen [2].The elastin is a protein that functions as a resistance to the formation of an aneurysm, whereas the collagen prevents rupture after the formulation of aneurysm occurs. Although this model showed the significance of collagen and elastin in the development and rupture of aneurysms which is very consistent with experimental observations, some assumptions were made in this paper, in order to improve the model of the CSF and perform an analysis of the resulting new 
model.

It must also be pointed out that modeling such biological multi-physics applications often require solution to coupled nonlinear partial differential equations describing the fluid-structure interaction (FSI). While it is possible to find exact solutions for simplified FSI problems either through linear approximations [24] or well-chosen boundary conditions [2], often these problems do not often admit exact solutions. One has to therefore resort to numerical methods such as finite difference methods [24] or finite element methods [23]. In [3] a computational model was developed that extended the work of [24] and the associated nonlinear system of coupled differential equations were solved numerically using implicit finite difference methods combined with Newton's method.

The outline of the paper is as follows. In Section 2, we introduce the mathematical model corresponding to the coupled fluid-structure interaction problem associated with the intracranial saccular aneurysm. Specifically, we first introduce the respective models for the CSF, blood flow and the arterial wall which are then combined to yield a coupled fluid structure interaction system that models the nonlinear elastodynamics problem. Section 3 presents a detailed analysis of the governing differential equation introduced in Section 2 that includes existence, uniqueness and analytical expressions for traveling wave solutions corresponding to linear and nonlinear cases respectively. In Section 4, we perform numerical simulation and computational experiments that help to validate our models. We finally conclude with some discussion and future work in Section 5.

\section{A MATHEMATICAL MODEL}

We consider the coupled interaction between the arterial wall, the blood flow within it and the CSF outside (See Figure 1). In this work we will assume radial symmetry and therefore we seek to develop a one-dimensional model along the x-direction as shown in the right panel in Figure 1. Note that while the simplification yields a 1D model, the associated analysis is quite involved which is the focus of the paper. Also, this simplified model helps to provide a further insight into the problem in higher dimensions. Next, we will develop 




Figure 1: Geometry of the coupled model

a model for each of the sections including the blood, wall and CSF.

\subsection{Model of Cerebral Spinal Fluid}

To model the flow of the CSF, we will consider the model employed in [24] where the assumption of radial symmetry allows us to consider Navier-Stokes equation to describe the flow of the CSF in one-dimension as follows:

$$
\rho v_{t}+\rho v v_{x}+P_{x}-\mu v_{x x}=F
$$

where $\rho$ is density, $v$ is velocity, $P$ is pressure, $\mu$ is viscosity, and $F$ is body force on the fluid. The natural external body forces $F$ include gravity which may be assumed to be negligible in comparison to the forces from the surrounding CSF and blood from within which will be incorporated in the model. Hence assuming that there is no external force $F$ acting in the fluid (2.1) becomes [24]:

$$
\rho v_{t}+\rho v v_{x}+P_{x}-\mu v_{x x}=0
$$

Here $x=0$ refers to the location of the outer wall (see Figure 1) which is left end boundary for the one-dimensional computational geometry considered for the CSF. As described in [24], the radial symmetry assumption justifies the validity of equations (2.1) and (2.2).

Next, we consider biologically motivated models for pressure gradients that will help to determine the pressure $P(x, t)$. The Womersley number is a dimensionless number that characterize the blood flow frequency and the Weissenberg number is a dimensionless number used to characterize viscoelastic flow. In [4], they considered models for pressure gradient that were either 
increasing and decreasing exponentially with time. They noted that for a fixed Womersley number and a different value of the Weissenberg number, the model for the pressure gradient decreasing with time result in a better approximation for volume flow rate. Assuming pressure gradient to be an exponentially decreasing function of time with a decay rate of $s$ and $P(0, t)=0$ we have [1]:

$$
P_{t}=-s P
$$

Next, we assume that the CSF is slightly compressible which yields the following equation of state [28]:

$$
\rho(P)=\rho_{0} e^{\gamma^{-1} P(x, t)}
$$

where $\gamma=\rho c^{2}$, and $\mathrm{c}$ is the speed of sound through the fluid. Taking the derivative of (2.4) with respect to $P$ gives:

$$
\frac{d \rho}{d P}=\left(\frac{\rho_{0}}{\gamma}\right) e^{\gamma^{-1} P(x, t)}
$$

Substituting (2.4) into (2.5) we obtain:

$$
\frac{d \rho}{d P}=\frac{\rho}{\gamma}
$$

The law of conservation of mass requires that the time derivative of the mass inside a control volume is equal to the mass flux across its boundaries. This may be stated as:

$$
\frac{\partial \rho}{\partial t}=-\frac{\partial}{\partial x}(\rho v)
$$

Applying the product and chain rule then gives:

$$
\frac{d \rho}{d P} \frac{\partial P}{\partial t}=-\rho \frac{\partial v}{\partial x}-v \frac{d \rho}{d P} \frac{\partial P}{\partial x}
$$

Substituting (2.6) into (2.7) gives:

$$
\frac{\rho}{\gamma} \frac{\partial P}{\partial t}=-\rho \frac{\partial v}{\partial x}-v \frac{\rho}{\gamma} \frac{\partial P}{\partial x}
$$

The second term in the right hand side is very small compared to the first term since $\gamma$ is very large and $\frac{\partial P}{\partial x}$ is exponentially decreasing which simplifies equation (2.8) to yield:

$$
\frac{\rho}{\gamma} \frac{\partial P}{\partial t}=-\rho \frac{\partial v}{\partial x}
$$


Then substituting (2.3) into (2.9) gives:

$$
P=\frac{\gamma}{s} v_{x}
$$

Using relation (2.10), our flow equation (2.2) becomes:

$$
\rho\left(v_{t}+v v_{x}\right)+\left(\frac{\gamma}{s}-\mu\right) v_{x x}=0
$$

Note that $\frac{\gamma}{s}-\mu>0$ based in the realistic values of these parameters [1].

There is evidence in the literature that CSF may be considered a nonNewtonian fluid. Therefore, one can enhance (2.11) to include these effects in the CSF model using a generalized power-law Bürger's equation for nonNewtonian fluids [26].

$$
\rho\left(v_{t}+v v_{x}\right)=\left(\mu-\frac{\gamma}{s}\right) \frac{\partial}{\partial x}\left(\left|\frac{\partial v}{\partial x}\right|^{n-1} \frac{\partial v}{\partial x}\right)
$$

In Section 3, we will prove the existence and uniqueness of the traveling wave solution to (2.12). Note that when $n=1$, we recover the Newtonian fluid model (2.11). Considering the case with $n=2$ (which is the focus of the paper), we will have the following fluid equation:

$$
\rho\left(v_{t}+v v_{x}\right)=\left(\mu-\frac{\gamma}{s}\right) \frac{\partial}{\partial x}\left(\left|\frac{\partial v}{\partial x}\right| \frac{\partial v}{\partial x}\right)
$$

\subsection{Model of Blood Pressure}

The blood pressure is modeled using Fourier series since the behavior of blood is observed to be pulsatile $[20,5,12]$. This relation may be described as:

$$
P_{B}(t)=P_{m}+\sum_{n=1}^{N}\left(A_{n} \cos (n w t)+B_{n} \sin (n w t)\right)
$$

where $P_{m}$ is the mean blood pressure, $A_{n}, B_{n}$ are Fourier coefficients, and $w$ is the fundamental circular frequency. Here $A_{n}, B_{n}, P_{m}, w$ are experimentally determined constants which are well-established for finite number of harmonics that approximates the blood pressure well $[5,12]$. 


\subsection{Model of Arterial Wall}

There are three main constituents of the artery wall, namely, the elastin, the collagen, and the smooth muscle $[11,7]$. Elastin and collagen are the main load bearers. The elastin is a stable protein and is considered the most load bearing element that functions as resistance to the formation of an aneurysm, whereas the collagen is the protein that is responsible for preventing rupture after formation of an aneurysm. The growth of the aneurysm is associated with deficiency of elastin and weakening of the artery wall [8]. Moreover, the mechanical contribution from the smooth muscle is not as prominent as the collagen and elastin [9]. The smooth muscle is made up of less than $10 \%$ of blood vessels which function to control and regulate the blood flow in the brain artery. Hence, in this work, we only incorporate elastin and collagen into the modeling of the arterial wall in order to obtain an accurate biological model of the aneurysm that can lead to better interpretation and prediction for this disease.

We consider the arterial wall to be modeled using a simple spring-mass system that incorporates the effects of elastin, collagen in the outer wall of the arteries. The force of this system may be denoted by $F_{S}$ which is given by $F_{O}-F_{I}$ where $F_{O}$ and $F_{I}$ are the forces of outer and inner wall respectively. This may be expressed as:

$$
F_{S}=K_{E} A_{E}(t) \sigma_{E}\left(\epsilon_{E}\right)+K_{C} A_{C}(t) \sigma_{C}\left(\epsilon_{C}\right)-a P_{B}(t)
$$

where $K_{E}, K_{C}$ are the scaling coefficients, $A_{E}(t)$ and $A_{C}(t)$ are the crosssectional areas, and $\sigma_{E}\left(\epsilon_{E}\right), \sigma_{C}\left(\epsilon_{C}\right)$ are the stresses for elastin and collagen respectively. In this work, we will employ the constitutive models for stress-strain to be materially linear and strain-displacement to be geometrically nonlinear [25]. This allows us to assume that the stresses are related to the respective strains linearly as:

$$
\sigma_{E}=\epsilon_{E}(t) \quad \sigma_{C}=\epsilon_{C}(t)
$$

and the strains to be related to the displacements non-linearly as:

$$
\epsilon_{E}=\left(((L+u(0, t)) / L)^{2}-1\right) / 2 \quad \epsilon_{C}=\left(\epsilon_{E}+\left(1-r^{2}\right) / 2\right) / r^{2}
$$


where $L$ denotes the length of the unstrained tissue, $u$ its extension, and $r$ is the stretched factor of unstrained tissue of collagen fiber. Note that $u(0, t)$ represents the displacement of the outer wall of the aneurysm. The focus of the entire work is on computing this physical quantity.

\subsection{Governing Equations of Motion}

In order to solve the system (2.13), we need two boundary conditions. The first boundary condition is at point $x=0$ (inner wall in Figure 1), and it can be derived from the model of blood pressure and the arterial wall that we have discussed. Note that the force balance equation at $x=0$ may be written as:

$$
F_{T}=F_{F}-F_{S}
$$

where $F_{T}=m v_{t}(0, t)$ is the product of the mass and acceleration, $F_{F}=$ $-\frac{\rho c^{2}}{s} v_{x}(0, t) a$, where $a$ is the cross-sectional area and $\rho$ the density of the $\mathrm{CSF}$.

Substituting (2.14) and (2.15) into (2.16) we obtain the following boundary condition at $x=0$ :

$$
\begin{aligned}
m v_{t}(0, t)=a P_{m} & -K_{E} A_{E}(t) \sigma_{E}\left(\epsilon_{E}\right)-K_{C} A_{C}(t) \sigma_{C}\left(\epsilon_{C}\right)-\frac{\rho c^{2}}{s} a v_{x}(0, t) \\
& +\sum_{n=1}^{N}\left(a A_{n} \cos (n w t)+a B_{n} \sin (n w t)\right)
\end{aligned}
$$

The second boundary condition can be obtained by using the plane wave approximation that states that the waves from the wall will die down some fixed distance away from the wall. If this can be applied at point $x=l$, then the second boundary condition becomes [24]:

$$
v(l, t)=-c u_{x}(l, t)
$$

Combining (2.12), (2.17) and (2.18), assuming the displacement and velocity start from rest, and substituting values of $\sigma_{E}, \sigma_{C}, \epsilon_{E}$, and $\epsilon_{C}$, we obtain the following system of coupled fluid-structure interaction equations:

$$
\rho\left(v_{t}+v v_{x}\right)=\left(\mu-\frac{\gamma}{s}\right) \frac{\partial}{\partial x}\left(\left|\frac{\partial v}{\partial x}\right|^{n-1} \frac{\partial v}{\partial x}\right)
$$




$$
\begin{aligned}
u_{t}= & v \\
u(x, 0)=v(x, 0)= & 0 \\
m v_{t}(0, t)= & a P_{B}(t)-K_{E C} u(0, t)-\frac{K_{E C}}{2 L} u^{2}(0, t) \\
& -\frac{\rho c^{2}}{s} a v_{x}(0, t)-\frac{K_{C} A_{C}\left(1-r^{2}\right)}{2 r^{2}} \\
v(l, t)= & -c u_{x}(l, t)
\end{aligned}
$$

where $K_{E C}=\left(\frac{K_{E} A_{E}}{L}+\frac{K_{C} A_{C}}{L r^{2}}\right)$. For simplicity, the cross-sectional areas of elastic and collagen are assumed to be constant. In particular, we consider $A_{E}(t)=A_{E}, A_{C}(t)=A_{C}$. The relation between strains and respective displacements is considered to be geometrically nonlinear, whereas the relation between stresses and strains is materially linear. The resulting system (2.19) is a coupled nonlinear fluid-structure interaction system whose solution would help to determine the displacement of the outer wall at $x=0$ given by $u(0, t)$. The latter provides a measure for rupture of the aneurysm. In the next section we will first prove the existence and uniqueness of the solution to (2.12). Following that we will describe how to obtain a traveling wave solution for both the linear case $(n=1)$ and the nonlinear case $(n=2)$ which are each respectively solved to obtain the displacement of the outer arterial wall $u(0, t)$.

\section{ANALYSIS OF THE MATHEMATICAL MODEL}

We will employ the Peano Existence and Uniqueness theorem in the implicit case $[21,13]$ to prove the following result.

Theorem 3.1. The traveling wave solution to the power law equation (2.12) exists and is unique.

Proof. Let us define $v(x, t)=v(\xi)=v(x-\lambda t)$. Then

$$
\frac{\partial v}{\partial x}=v^{\prime}(\xi) \quad \frac{\partial v}{\partial t}=-\lambda v^{\prime}(\xi)
$$

Substituting 3.1 in (2.12), we obtain the following:

$$
\rho\left(-\lambda v^{\prime}(\xi)+v(\xi) v^{\prime}(\xi)\right)=\rho\left(-\lambda v(\xi)+\frac{1}{2} v(\xi)^{2}\right)^{\prime}
$$




$$
=\left(\mu-\frac{\gamma}{s}\right)\left(\left|v^{\prime}(\xi)\right|^{n-1} v^{\prime}(\xi)\right)^{\prime}
$$

Integrating the above equation with respect to $\xi$ we obtain:

$$
\rho\left(-\lambda v(\xi)+\frac{1}{2} v(\xi)^{2}\right)=\left(\mu-\frac{\gamma}{s}\right)\left(\left|v^{\prime}(\xi)\right|^{n-1} v^{\prime}(\xi)\right)+C_{1}
$$

where $C_{1}$ is constant.

Let us now define

$$
F\left(v^{\prime}(\xi), v(\xi), \xi\right)=-\left(\mu-\frac{\gamma}{s}\right)\left(\left|v^{\prime}(\xi)\right|^{n-1} v^{\prime}(\xi)\right)+\rho\left(-\lambda v(\xi)+\frac{1}{2} v(\xi)^{2}\right)-C_{1}
$$

In order to prove existence and uniqueness, we will show the following:

1. There exist an open region $\mathfrak{U}$ of 3 -dimensional Euclidean space such that $F\left(v^{\prime}(\xi), v(\xi), \xi\right)$ is defined and continuous on $\mathfrak{U}$.

2. $\frac{\partial F}{\partial v^{\prime}}$ exists and is continuous on $\mathfrak{U}$

3. $\frac{\partial F}{\partial v}$ exists and is continuous on $\mathfrak{U}$.

4. There exists a point $\left(v_{0}^{\prime}, v_{0}, \xi_{0}\right)$ in $\mathfrak{U}$ such that $F\left(v_{0}^{\prime}, v_{0}, \xi_{0}\right)=0$ and $J=\frac{\partial F}{\partial v^{\prime}} \neq 0$ at this point. Here $J$ refers to the standard Jacobian matrix which consists of all first-order partial derivatives for a vectorvalued function $F\left(v_{1}^{\prime}, v_{2}^{\prime}, \ldots, v_{n}^{\prime}\right)$. Note that in our case, $n=1$.

We will now substantiate each of the items above.

Remark 3.2. The open region $\mathfrak{U}$ depends on the power of $n$ in the expression of $F$. Since we are only dealing with the case $n=1$ and $n=2$ in this paper, $\mathfrak{U}$ is the whole 3 -dimensional Euclidean space.

Through this remark we satisfy item (1). Assuming $v^{\prime}>0$ and taking the partial derivative of (3.4) with respect to $v^{\prime}$ we have:

$$
\frac{\partial F}{\partial v^{\prime}}=-\left(\mu-\frac{\gamma}{s}\right) n\left(v^{\prime}\right)^{n-1}
$$

It is clear from (3.5) that $\frac{\partial F}{\partial v^{\prime}} \neq 0$ and is continuous. This satisfies item (2). 
Differentiating (3.4) with respect to the velocity $v$, we get,

$$
\frac{\partial F}{\partial v}=-\rho \lambda+\rho v
$$

which is also continuous. This satisfies item (3).

To satisfy item (4), note that at the particular point

$$
\left(v_{0}^{\prime}, v_{0}, \xi_{0}\right)=\left(\left(\frac{\rho \lambda^{2}}{2\left(\frac{\gamma}{s}-\mu\right)}\right)^{\frac{1}{n}}, \lambda, \xi_{0}\right)
$$

setting $C_{1}=0$ results in $F\left(v_{0}^{\prime}, v_{0}, \xi_{0}\right)=0$ and $\frac{\partial F}{\partial v^{\prime}} \neq 0$.

This confirms the existence and uniqueness according to the Peano theorem in the implicit case $([13]$, p. 28-31) and the uniqueness in the implicit case ([13], p.44-47) for the power law Burger's equation.

Now that we have proved uniqueness and existence of the solution, next we try to compute this traveling wave solution for two cases. First we consider the case of the CSF having negligible nonlinear effects. The second involves inclusion of nonlinear effects.

\subsection{The Traveling Wave Solution: Linear Model}

Let us consider the case $n=1$ with a negligible nonlinear effect. Then, the system above (2.19) becomes

$$
\begin{aligned}
\rho v_{t} & =\left(\mu-\frac{\gamma}{s}\right) v_{x x}, \\
u_{t} & =v \\
u(x, 0)=v(x, 0) & =0 \\
m v_{t}(0, t) & =a P_{B}(t)-D_{1} u(0, t)-D_{2} u^{2}(0, t) \\
& -\frac{\rho c^{2}}{s} a v_{x}(0, t)-D_{3}, \\
v(l, t)= & -c u_{x}(l, t) .
\end{aligned}
$$

where

$$
\begin{aligned}
& D_{1}=\frac{k_{E} A_{E}}{L}+\frac{k_{C} A_{C}}{L r^{2}}, \\
& D_{2}=\frac{k_{E} A_{E}}{2 L^{2}}+\frac{k_{C} A_{C}}{2 L^{2} r^{2}},
\end{aligned}
$$




$$
D_{3}=\frac{k_{C} A_{C}\left(1-r^{2}\right)}{2 r^{2}} .
$$

The following theorem shows how to compute the displacement of the outer wall of the aneurysm $u(0, t)$.

Theorem 3.3. The displacement $u(0, t)$ in (3.7) is given by:

$$
u(0, t)=\frac{-D_{1}+\sqrt{D_{1}^{2}-4 D_{2}\left(G(t)-a P_{B}(t)+D_{3}\right)}}{2 D_{2}}
$$

where

$$
G(t)=\left(a P_{m}+a \sum_{n=1}^{N} A_{n}-D_{3}\right) e^{\frac{\lambda^{2} \rho}{\mu-\frac{\gamma}{s}} t}
$$

Proof. To find a traveling wave solution for the governing equation of the system (3.7), we assume that the solution will be of the form:

$$
v(x, t)=f(\xi)
$$

where $\xi=x-\lambda t$. Then

$$
\begin{aligned}
& v_{t}=-\lambda f^{\prime}(\xi) \\
& v_{x}=f^{\prime}(\xi)
\end{aligned}
$$

Substituting (3.9) in the governing equation of system (3.7), we obtain:

$$
f^{\prime \prime}(\xi)+\frac{\lambda \rho}{\mu-\frac{\gamma}{s}} f^{\prime}(\xi)=0 .
$$

Integrating (3.10) once with respect to $\xi$ yields:

$$
f^{\prime}(\xi)+\frac{\lambda \rho}{\mu-\frac{\gamma}{s}} f(\xi)=C_{1},
$$

where $C_{1}$ is constant to be determined. Solving equation (3.11), we obtain the following traveling wave solution

$$
f(\xi)=C_{1} \frac{\mu-\frac{\gamma}{s}}{\lambda \rho}+C_{2} e^{-\frac{\lambda \rho}{\mu-\frac{\gamma}{s} \xi} .}
$$

Using $v(0,0)=f(0)=0$, we obtain:

$$
C_{1}=\frac{-\lambda \rho}{\left(\mu-\frac{\gamma}{s}\right)} C_{2} .
$$


In order to find the solution $u(0, t)$, we find $v_{t}(0, t)$ and $v_{x}(0, t)$ from $(3.12)$, substitute them in the first boundary condition of system (3.7) and solve for $u(0, t)$. These derivatives may be computed as:

$$
\begin{aligned}
& v_{t}(0, t)=\frac{\rho \lambda^{2}}{\mu-\frac{\gamma}{s}} C_{2} e^{\frac{\lambda^{2} \rho}{\mu-\frac{\gamma}{s}} t}, \\
& v_{x}(0, t)=-\frac{\rho \lambda}{\mu-\frac{\gamma}{s}} C_{2} e^{\frac{\lambda^{2} \rho}{\mu-\frac{\gamma}{s}} t} .
\end{aligned}
$$

Then the physically meaningful solution is computed as:

$$
u(0, t)=\frac{-D_{1}+\sqrt{D_{1}^{2}-4 D_{2}\left(G(t)-a P_{B}(t)+D_{3}\right)}}{2 D_{2}}
$$

where

$$
\begin{aligned}
G(t) & =m v_{t}(0, t)+\frac{\rho c^{2} a}{s} v_{x}(0, t) \\
& =\frac{\rho \lambda}{\mu-\frac{\gamma}{s}} C_{2} e^{\frac{\lambda^{2} \rho}{\mu-\frac{\gamma}{s}} t}\left(m \lambda-\frac{\rho c^{2} a}{s}\right)
\end{aligned}
$$

Note we have to make the following restriction in order to have a real root.

$$
\mu-\frac{\gamma}{s}<0
$$

This assumption makes physical sense for the values of the parameters.

To obtain $C_{2}$, we use the first initial condition in $(3.7) u(0,0)=0$ to yield:

$$
\begin{array}{r}
-D_{1}+\sqrt{D_{1}^{2}-4 D_{2}\left(G(0)-a P_{B}(0)+D_{3}\right)}=0 \\
\Longrightarrow G(0)-a P_{B}(0)+D_{3}=0
\end{array}
$$

Substituting $G(0), P_{B}(0)=a P_{m}+a \sum_{n=1}^{N} A_{n}$ now yields:

$$
\frac{\rho \lambda}{\mu-\frac{\gamma}{s}} C_{2}\left(m \lambda-\frac{\rho c^{2} a}{s}\right)-a P_{m}-a \sum_{n=1}^{N} A_{n}+D_{3}=0 .
$$

Solving the above equation for $C_{2}$ gives

$$
C_{2}=\frac{\left(a P_{m}+a \sum_{n=1}^{N} A_{n}-D_{3}\right)}{\frac{\rho \lambda}{\mu-\frac{\gamma}{s}}\left(m \lambda-\frac{\rho c^{2} a}{s}\right)} .
$$


Finally, to find $\lambda$, we use (3.8) and the second boundary condition in (3.7). Using the relation between displacement and velocity $u_{t}(x, t)=v(x, t)$ and (3.8) we get

$$
u_{t}(x, t)=f(x-\lambda t)
$$

Assuming,

$$
f(x-\lambda t)=\frac{d H(\xi)}{d \xi}
$$

we get,

$$
u_{t}(x, t)=\frac{d H(\xi)}{d \xi}
$$

Integrating this relation with respect to $t$ gives:

$$
u(x, t)=-\frac{1}{\lambda} H(x-\lambda t) .
$$

Differentiating with respect to $x$ yields:

$$
u_{x}(x, t)=-\frac{1}{\lambda} f(x-\lambda t) .
$$

Substituting this into the second boundary condition of the system (3.7) at $x=l$ we obtain:

$$
\begin{aligned}
v(l, t) & =-c u_{x}(l, t) \\
f(l-\lambda t) & =-c \frac{-1}{\lambda} f(l-\lambda t) \\
\Longrightarrow \lambda & =c
\end{aligned}
$$

Substituting $C_{1}, C_{2}, \lambda$ into (3.16) we can obtain $u(0, t)$.

\subsection{The Traveling Wave Solution: Nonlinear Power Law Model}

Considering $n=2$ in the system (2.19), we obtain the following system

$$
\begin{aligned}
\rho\left(v_{t}+v v_{x}\right) & =\left(\mu-\frac{\gamma}{s}\right) \frac{\partial}{\partial x}\left(\left|\frac{\partial v}{\partial x}\right| \frac{\partial v}{\partial x}\right), \\
u_{t} & =v \\
u(x, 0)=v(x, 0) & =0, \\
m v_{t}(0, t) & =a P_{B}(t)-D_{1} u(0, t)-D_{2} u^{2}(0, t)
\end{aligned}
$$




$$
\begin{aligned}
& -\frac{\rho c^{2}}{s} a v_{x}(0, t)-D_{3} \\
v(l, t)=- & -c u_{x}(l, t) .
\end{aligned}
$$

To find a traveling wave solution for system (3.20), we assume that the solution will be in the form:

$$
v(x, t)=f(\xi)
$$

where $\xi=x-\lambda t$.

Substituting (3.21) in the governing equation of system (3.20), we obtain:

$$
-\lambda f^{\prime}(\xi)+f(\xi) f^{\prime}(\xi)-\frac{1}{\rho}\left(\mu-\frac{\gamma}{s}\right)\left(\left|f^{\prime}\right| f^{\prime}\right)^{\prime}=0 .
$$

Note that the displacement of the outer wall of the aneurysm $u(0, t)$ depends on $f^{\prime}(\xi)$. In the next two theorems, we will derive the solutions for two different cases of $f^{\prime}(\xi)$ being negative and positive respectively. Let us define

$$
q=\sqrt{\frac{\rho}{2\left(\frac{\gamma}{s}-\mu\right)}}
$$

Remark 3.4. Since $f^{\prime}(\xi)=-\frac{v_{t}}{\lambda}=v_{x}, f^{\prime}(\xi)<0$ when $v_{t}>0$ and $v_{x}<0$. Likewise, $f^{\prime}(\xi)>0$ when $v_{t}<0$ and $v_{x}>0$. The quantity $q$ is related to (3.22) which we will use later when we solve the equation (3.23) to get (3.24) and when we solve the equation (3.28) to get (3.29) in the proofs of theorem 3.5 and theorem 3.6.

Theorem 3.5. If $f^{\prime}(\xi)<0$ then the displacement $u(0, t)$ that satisfies (3.20) is given by:

$$
u(0, t)=\frac{-D_{1}+\sqrt{D_{1}^{2}-4 D_{2}\left(G(t)-a P_{B}(t)+D_{3}\right)}}{2 D_{2}},
$$

where

$$
G(t)=q\left(-m \lambda+\frac{\rho c^{2} a}{s}\right)\left(C_{1} e^{-\lambda q t}-C_{2} e^{\lambda q t}\right) .
$$

and

$$
C_{1}=\frac{1}{2}\left(\begin{array}{c}
a P_{m}+a \sum_{n=1}^{N} A_{n}-D_{3} \\
\left.-\lambda+\frac{\rho c^{2} a}{s}\right)
\end{array}\right)
$$




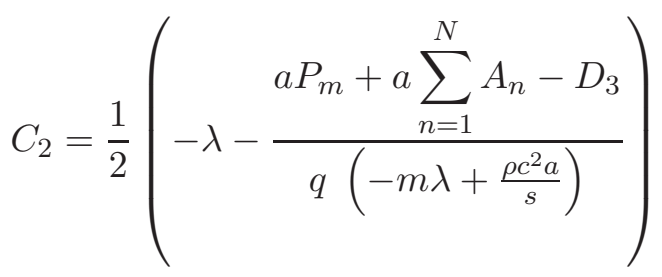

Note that the solution (3.25) is not physically valid and is exponentially unbounded.

Proof. Since $f^{\prime}(\xi)<0$, equation (3.22) becomes,

$$
\begin{aligned}
-\lambda f^{\prime}(\xi)+f(\xi) f^{\prime}(\xi)-\frac{1}{\rho}\left(\mu-\frac{\gamma}{s}\right)\left(-f^{\prime 2}\right)^{\prime} & =0 \\
\Longrightarrow-\lambda f^{\prime}(\xi)+f(\xi) f^{\prime}(\xi)-\frac{1}{\rho}\left(\mu-\frac{\gamma}{s}\right)\left(-2 f^{\prime} f^{\prime \prime}\right) & =0 .
\end{aligned}
$$

The above equation can be simplified as

$$
f^{\prime \prime}(\xi)+\frac{\rho}{2\left(\mu-\frac{\gamma}{s}\right)} f(\xi)-\frac{\rho}{2\left(\mu-\frac{\gamma}{s}\right)} \lambda=0 .
$$

Since we have $\mu-\frac{\gamma}{s}<0$, the solution to above equation is

$$
f(\xi)=C_{1} e^{q \xi}+C_{2} e^{-q \xi}+\lambda
$$

Differentiating equation (3.24) with respect to the $t$ and $x$ variables respectively, yields:

$$
\begin{aligned}
& v_{t}(x, t)=-\lambda f^{\prime}(\xi)=-\lambda q\left[C_{1} e^{q \xi}-C_{2} e^{-q \xi}\right], \\
& v_{x}(x, t)=f^{\prime}(\xi)=q\left[C_{1} e^{q \xi}-C_{2} e^{-q \xi}\right] .
\end{aligned}
$$

Substituting $v_{t}(0, t)$ and $v_{x}(0, t)$ into the first boundary condition of the system $(3.20)$ in order to find $u(0, t)$ :

$$
u(0, t)=\frac{-D_{1}+\sqrt{D_{1}^{2}-4 D_{2}\left(G(t)-a P_{B}(t)+D_{3}\right)}}{2 D_{2}},
$$

where

$$
\begin{aligned}
G(t) & =m v_{t}(0, t)+\frac{\rho c^{2} a}{s} v_{x}(0, t) \\
& =q\left(-m \lambda+\frac{\rho c^{2} a}{s}\right)\left[C_{1} e^{-\lambda q t}-C_{2} e^{\lambda q t}\right] .
\end{aligned}
$$


In order to find the coefficients $C_{1}$ and $C_{2}$, we will use the initial conditions of system (3.20) into (3.24) and (3.25) that yields the system of equations:

$$
\begin{aligned}
C_{1}+C_{2}+\lambda & =0, \\
C_{1}-C_{2}-\frac{\left(a P_{m}+a \sum_{n=1}^{N} A_{n}-D_{3}\right)}{q\left(-m \lambda+\frac{\rho c^{2} a}{s}\right)} & =0 .
\end{aligned}
$$

Solving this system of equations we obtain:

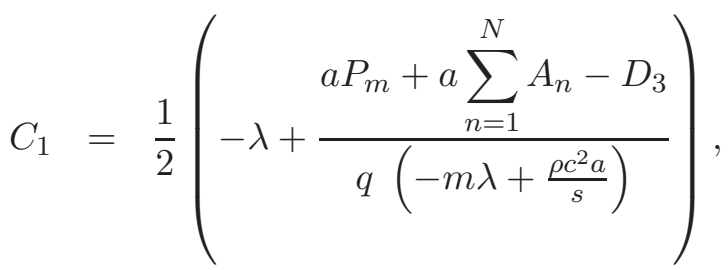

$$
\begin{aligned}
& C_{2}=\frac{1}{2}\left(-\lambda-\frac{a P_{m}+a \sum_{n=1}^{N} A_{n}-D_{3}}{q\left(-m \lambda+\frac{\rho c^{2} a}{s}\right)}\right) .
\end{aligned}
$$

Theorem 3.6. If $f^{\prime}(\xi)>0$ then the displacement $u(0, t)$ that satisfies (3.20) is given by:

$$
u(0, t)=\frac{-D_{1}+\sqrt{D_{1}^{2}-4 D_{2}\left(G(t)-a P_{B}(t)+D_{3}\right)}}{2 D_{2}},
$$

where

$$
G(t)=q\left(-m \lambda+\frac{\rho c^{2} a}{s}\right)\left[C_{1} \sin (q \lambda t)+C_{2} \cos (q \lambda t)\right] .
$$

and

$$
C_{1}=-\lambda \quad C_{2}=\frac{a P_{m}+a \sum_{n=1}^{N} A_{n}-D_{3}}{q\left(-m \lambda+\frac{\rho c^{2} a}{s}\right)} .
$$

Proof. From (3.22) we have,

$$
-\lambda f^{\prime}(\xi)+f(\xi) f^{\prime}(\xi)-\frac{1}{\rho}\left(\mu-\frac{\gamma}{s}\right)\left(f^{\prime 2}\right)^{\prime}=0,
$$




$$
\Longrightarrow-\lambda f^{\prime}(\xi)+f(\xi) f^{\prime}(\xi)-\frac{1}{\rho}\left(\mu-\frac{\gamma}{s}\right)\left(2 f^{\prime} f^{\prime \prime}\right)=0 .
$$

Simplifying this yields

$$
f^{\prime \prime}(\xi)-\frac{\rho}{2\left(\mu-\frac{\gamma}{s}\right)} f(\xi)+\frac{\rho}{2\left(\mu-\frac{\gamma}{s}\right)} \lambda=0 .
$$

Since $\mu-\frac{\gamma}{s}<0$, then the solution to the equation is

$$
f(\xi)=C_{1} \cos (q \xi)+C_{2} \sin (q \xi)+\lambda
$$

We find $v_{t}(x, t)$ and $v_{x}(x, t)$ by differentiating above solution (3.29) with respect to $t$ and $x$ :

$$
\begin{aligned}
& v_{t}(x, t)=-\lambda f^{\prime}(\xi)=-\lambda q\left[-C_{1} \sin (q \xi)+C_{2} \cos (q \xi)\right], \\
& v_{x}(x, t)=f^{\prime}(\xi)=q\left[-C_{1} \sin (q \xi)+C_{2} \cos (q \xi)\right]
\end{aligned}
$$

Then we substitute $v_{t}(0, t)$ and $v_{x}(0, t)$ into the first boundary condition of system (3.20) to obtain a solution for $u(0, t)$

$$
u(0, t)=\frac{-D_{1}+\sqrt{D_{1}^{2}-4 D_{2}\left(G(t)-a P_{B}(t)+D_{3}\right)}}{2 D_{2}},
$$

where

$$
\begin{aligned}
G(t) & =m v_{t}(0, t)+\frac{\rho c^{2} a}{s} v_{x}(0, t) \\
& =q\left(-m \lambda+\frac{\rho c^{2} a}{s}\right)\left[C_{1} \sin (q \lambda t)+C_{2} \cos (q \lambda t)\right]
\end{aligned}
$$

Note that we also have the following restriction in order to have a real valued solution.

$$
D_{1}^{2}-4 D_{2}\left(G(t)-a P_{B}(t)+D_{3}\right) \geq 0 .
$$

The restriction above can be simplified as

$$
\begin{aligned}
\frac{D_{1}^{2}}{4 D_{2}}-D_{3}+a P_{B}(t) & \geq G(t) \\
\Longrightarrow \frac{K_{E} A_{E}+K_{C} A_{C}}{2}+a P_{B}(t) & \geq G(t)
\end{aligned}
$$

Remark 3.7. Equation (3.33) also shows that $G(t)-a P_{B}(t)$ must be bounded by the average of $K_{E} A_{E}$ and $K_{C} A_{C}$. 
In order to find the coefficients $C_{1}$ and $C_{2}$, we will substitute the initial conditions of systems (3.20) into equations (3.29) and (3.31) to obtains

$$
\begin{aligned}
C_{1} & =-\lambda, \\
C_{2} & =\frac{a P_{m}+a \sum_{n=1}^{N} A_{n}-D_{3}}{q\left(-m \lambda+\frac{\rho c^{2} a}{s}\right)} .
\end{aligned}
$$

Note that the solution to (3.31) is bounded and therefore physically meaningful.

\section{COMPUTATIONAL EXPERIMENTS}

For our computational experiments, we use the following realistic values from [24]. For the CSF, $\rho=1000 \mathrm{~kg} / \mathrm{m}^{3}, c=1500 \mathrm{~m} / \mathrm{s}, \mu=0.000126 \mathrm{Ns} / \mathrm{m}^{2}, \mathrm{~s}=$ 0.5 are used. For the aneurysmal wall we use, $a=0.035 e-06, K_{E}=97.6 e+01$ $k P a, K_{C}=4.40 \mathrm{kPa}, A_{E}=0.0006 \mathrm{~m}^{2}, A_{C}=0.000001 \mathrm{~m}^{2}, r=0.006 \mathrm{~m}$, $m=0.001 \mathrm{~kg}$, and $L=0.0009 \mathrm{~m}$ are used. Finally, $P_{m}=8759.279403 \mathrm{mmHg}$, $w=1 \mathrm{rad} / \mathrm{s}$ are used for the blood pressure model along with the harmonics, $A_{1}=-7.13, A_{2}=-3.08, A_{3}=-0.130, A_{4}=-0.205, A_{5}=0.0662, B_{1}=$ $4.64, B_{2}=-1.18, B_{3}=-0.564, B_{4}=-0.346, B_{5}=-0.120$, all in $\mathrm{mmHg}$ (see [24] and references therein). Note that these values are physically meaningful and meet both restrictions (3.17) and (3.33).

First, we perform experiments to illustrate the influence of the displacement of the outer wall $u(0, t)$ on the stiffness of the elastin and collagen respectively. In figure (2), we plot the displacement for increasing values of $K_{E}$ for the linear model (3.16) when the value of the stiffness of collagen is kept constant. One must expect that as the stiffness increases it will be harder to displace the outer wall in the interaction. This observation is confirmed in the graph. Next, we studied the influence of the stiffness of elastin on the displacement of the outer wall $u(0, t)$ for the nonlinear power-law model (3.31). Figure (3) illustrates this. We observe the same behaviour as noted in the linear case, which is that when the stiffness increases the displacement decreases. 




Figure 2: Influence of $K_{E}$ on the linear model (3.16)

However, we also note that the magnitude of the displacement in comparison to the linear model in Figure (2) is significantly different suggesting that the nonlinear effects must be taken into account when modeling. Finally, to study the influence of the stiffness of collagen, we ran an experiment keeping the value of stiffness of elastin constant and increasing the value of collagen. From Figures 4 and 5, we note that as $K_{C}$ increases, the amplitude for both the linear model (3.16) and nonlinear power law model (3.31) increases but the magnitude and behaviour of the solutions are significantly different.

\section{CONCLUSION AND FUTURE WORK}

In this work, a new mathematical model was developed that describes the nonlinear elastodynamics of an aneurysm interacting with blood flow and CSF. While the model was formulated as a one-dimensional model using an assumption of radial symmetry, the nonlinear features incorporated therein, such as 


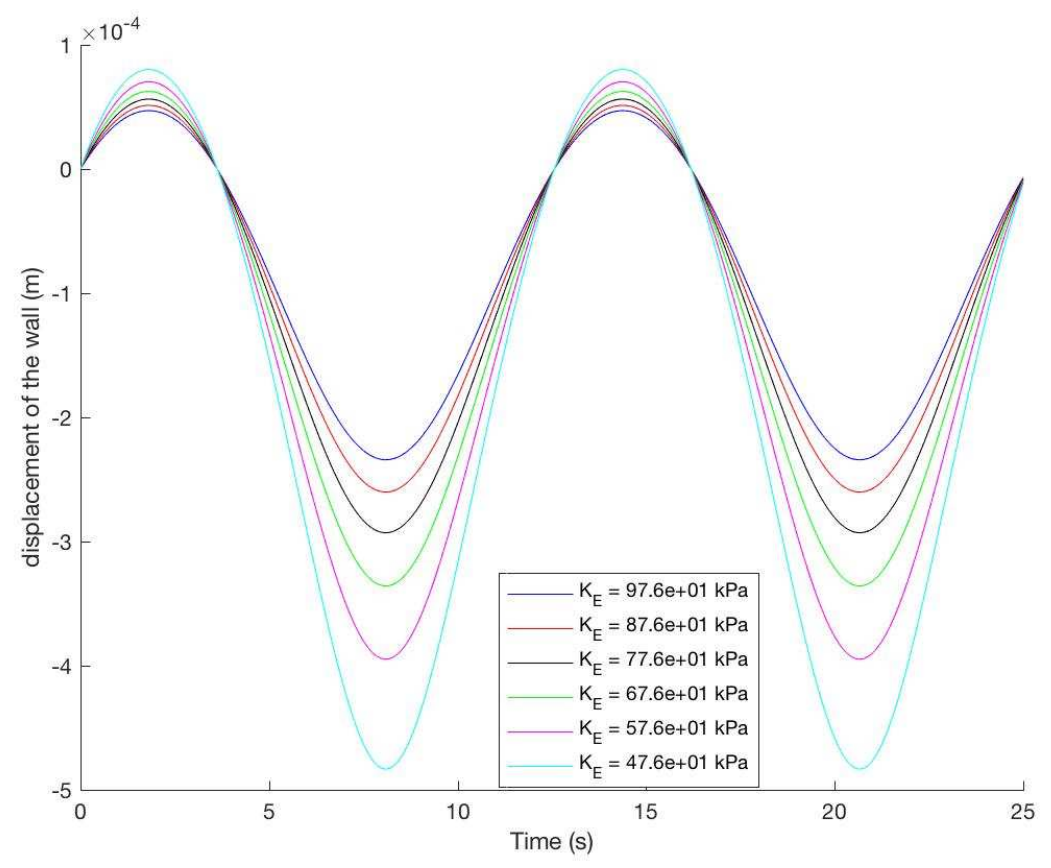

Figure 3: Influence of $K_{E}$ on nonlinear power law model (3.31)

power-law for fluid velocity and geometric nonlinearity of the wall make the associated model very challenging. The focus of this work was on the analysis of the associated governing differential equations. Specifically, we were able to employ sophisticated tools such as the Peano's theorems to prove existence and uniqueness of the solution to the governing differential equations. We were also able to derive an exact expressions for the displacement of the outer wall of the aneurysm $u(0, t)$ using traveling wave solution formulation. Finally, our numerical results validated our expected behavior of the displacement $u(0, t)$ as a function of the stiffness of elastin $K_{E}$ and collagen $K_{C}$ which substantiated with known biological behavior.

However, there is an opportunity to enhance the model presented in this work in several ways. First, it is well-known that the wall is viscoelastic and therefore one can consider modifying the stress-strain relations accordingly. One may also consider introducing hyperelastic constitutive models that are more nonlinear. This study compared the linear case $(n=1)$ with the nonlinear case of $(n=2)$. One can try to extend this study to any positive real value 


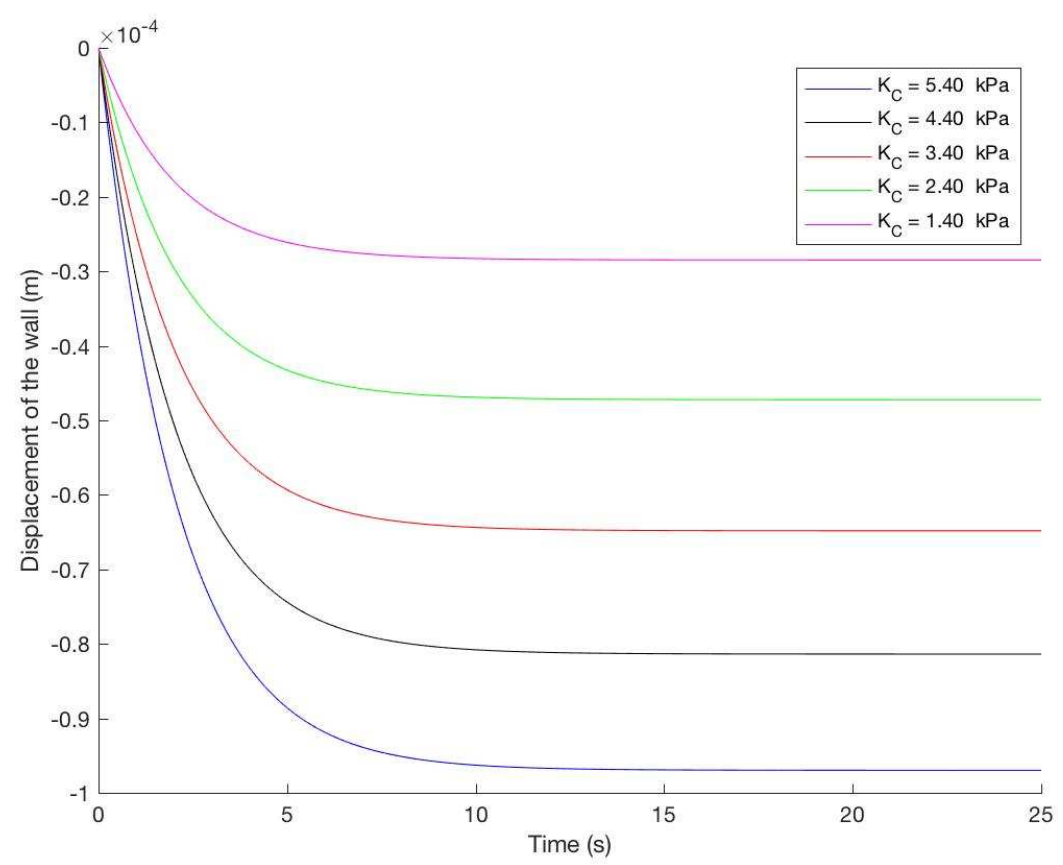

Figure 4: Influence of $K_{C}$ on the linear model (3.16)

of $n$ which is a challenging problem. Finally, one can also consider extending this work to higher dimensions. We hope to consider these enhancements in our forthcoming papers.

\section{REFERENCES}

[1] M. A. Azad, L. S. Andallah, An analytical Solution of 1D Navier-Stokes Equation, International Journal of Scientific and Engineering Research $\mathbf{5}$ 2 (2014), 1342- 1348.

[2] M. Badgaish, J. Lin, and P. Seshaiyer, Nonlinear Dynamics and Analysis of Intracranial Saccular Aneurysm with Growth and Remodeling, Journal of Nonlinear Dynamics 2016 (2016), Article ID 2869083, doi:10.1155/2016/2869083.

[3] M. Badgaish and P. Seshaiyer, Modeling, Computation and Simulation 


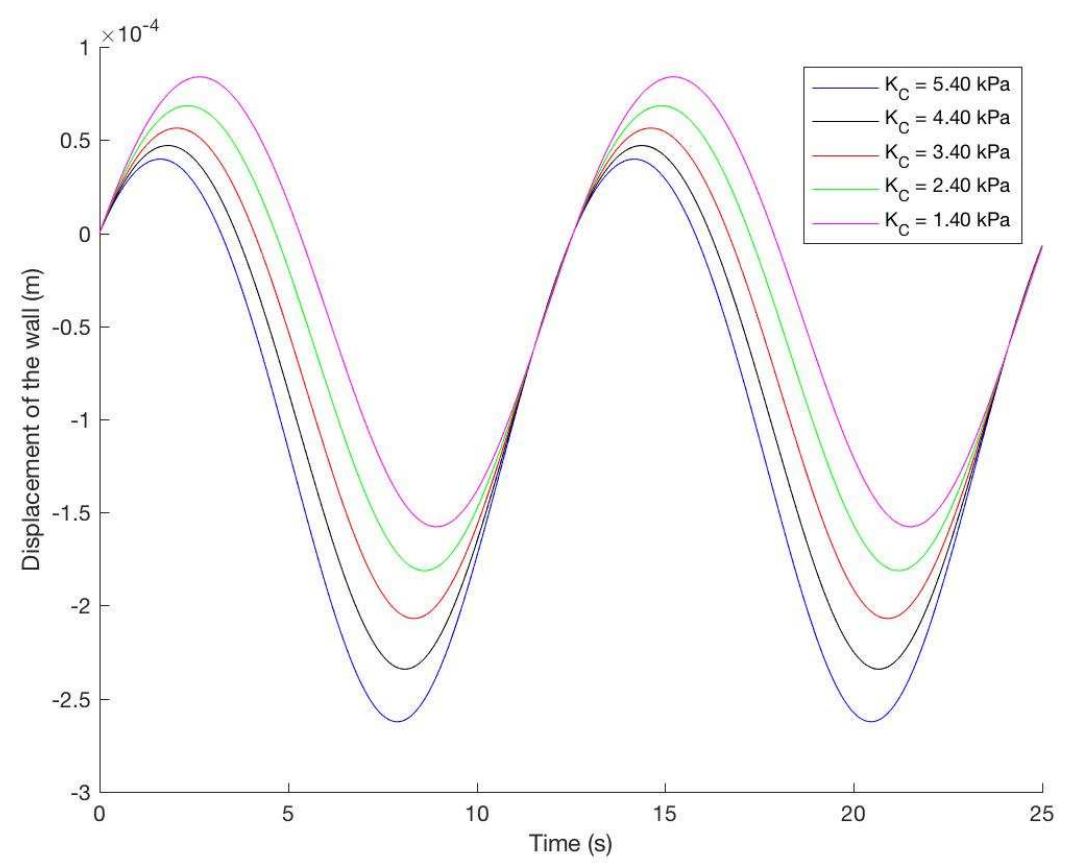

Figure 5: Influence of $K_{C}$ on the nonlinear power law model (3.31)

of nonlinear soft tissue interaction with flow dynamics with applications to aneurysms, International Journal of Novel Ideas, Accepted (2017).

[4] F. Carapau, and A. sequeria,Axisymmetric motion of second order viscous fluid in circular straight tube under pressure gradients varying exponentially with time, WIT Transactions on Engineering Sciences 52, 2006.

[5] G.G. Ferguson, Direct Measurment of Mean and Pulsatile Blood Pressure at Operation in Humman Intracranial Saccular Aneurysms, Journal of Neurosurgery 36 (1972), 560-563.

[6] A. Foster, D. Anderson and P. Seshaiyer, Numerical Modeling and Analysis of Fluid Structure Interaction in Application to Cerebral Arteries, GMU Review 20 (2011), 62-73.

[7] P. Fratzl, Collagen: Structure and Mechanics, Springer, 2008.

[8] C.M. He, and M. Roach, The Composition and Mechanical Properties of Abdominal Aortic Aneurysms, J Vasc Surg 20 (1) (1993) , 6-13. 
[9] G.A. Holzapfel, T.C. Gasser, R.W. Ogden, A New Constitutive framework for arterial wall mechanics and a comparative study of material models. J Elasticity 61 (2000), 1-48.

[10] J.J. Jain, Mechanism of Rupture in Interacrinal Anueurysms, Surgery 54 (1963), 347-350.

[11] P. Lanzer, and E. Topal (2002),Pan Vascular Medicine: Integrated Clinical Management, Springer, 2002.

[12] W.R. Milnor,Hemodynamics, Williams and Wilkens,Baltimore,1982.

[13] F.J. Murray, K. S. Miller, Existence Theorems for Ordinary Differential Equations, Washington Square, New YORK, New York Uni. Press, 1954.

[14] M.L. Raghavan, D.A. Vorp , Towards a Biomechanical Tool to Evaluate Rupture Potential of Abdominal Aortic Aneurysms:Identification of a finite strain constitutive model and evalution of its applicability, J.Biomech. 33 (2000), 475-482.

[15] A. Samuelson and P. Seshaiyer,Stability of Membrane Elastodynamics with applications to Cylindrical Aneurysms, Journal of Applied Mathematics doi:10.1155/2011/906475 (2011).

[16] L.N. Sekhar, R.J. Sclabassi, M. Sun, H.B. Blue, J.F. Wasserman, Intraaneurysmal Pressure measurements in Experimental Saccular Aneurysms in Dogs, Stroke 19 (1988), 352-356.

[17] P. Seshaiyer and Jay D. Humphrey , On the protective role of contact constraints in saccular aneurysms, Journal of Biomechanics 34 (2001), 607-612.

[18] P. Seshaiyer, F. P. K. Hsu, A. D. Shah, S. K. Kyriacou and J. D. Humphrey, Multiaxial mechanical behavior of human saccular aneurysms, Computer Methods in Biomechanics and Biomedical Engineering 4 (2001), 281-289.

[19] P. Seshaiyer, J.D. Humphrey, A sub-domain inverse finite element characterization of hyper elastic membranes including soft tissues, J. of Biomech.Engg.125 3 (2003), 363-371. 
[20] A.D. Shah and J.D. Humphrey, Finite Strain Elastodynamics of Intracranial Saccular Aneurysms, Journal Of Biomechanics 32 (1999), 593-599.

[21] Y. Shu, Numerical Solution of generalized Burgers' Equation for Some Incompressible Non-Newtonian Fluid, University of New Orlean Theses and Dissertation 2051(2015).

[22] T.E. Simkins, W.E. Stehbens, Vibrational Behavior of Arterial Aneurysms, Letters in Applied and Engineering Sciences 1 (1973), 85100.

[23] E.W. Swim and P. Seshaiyer (2006). A nonconforming finite element method for fluidstructure interaction problems. Computer Methods in Applied Mechanics and Engineering, 195(17), 2088-2099.

[24] S. Venuti and P. Seshaiyer, Modeling, Analysis and Computation of Fluidstructure interaction models for biological systems, SIAM Undergrad. Research Online $\mathbf{3}$ (2010), 1-17.

[25] P. Watton, N. Hill,and M. Heil, A Mathematical Model for the Growth of the Abdominal Aortic Aneurysm,Biomech. Model. Mechanobiology 32 (2004), 98-133.

[26] D. Wei and H. Borden, Traveling Wave Solution of Burger's Equation for Power-Law Non-Newtonian Flows, Appl. Math. E-Notes 11 (2011), $133-138$.

[27] T.B.M. Wilmink, C.R.G. Quick,C.S. Hubbard,N.E. Day, The Influence of Screening on the Incidence of Ruptured Abdominal aortic Aneurysms, J. Vasc surg 302 (1999), 203-208.

[28] W. Zimmerman, Flow in Porous Media, Department of Earth Science and Engineering, Imperial Collage, UK, 2002. 
